\title{
Increased vascular endothelial growth factor expression predicts a worse prognosis for laryngeal cancer patients: a meta-analysis
}

\author{
L-P ZHANG ${ }^{1}$, H-L CHEN ${ }^{2}$ \\ ${ }^{1}$ Department of Otolaryngology Head and Neck Surgery, Affiliated Hospital of Nantong University, PR China, \\ ${ }^{2}$ School of Nursing, Nantong University, PR China
}

\begin{abstract}
Objective: This study assessed the relationship between vascular endothelial growth factor expression and the laryngeal cancer prognosis.

Methods: Systematic computerised searches of PubMed were performed up to 31 January 2015. Prognostic endpoints were overall survival and disease-free survival. The pooled hazard ratios for overall survival and disease-free survival were also calculated.

Results: Seven studies containing 975 patients were included. The pooled hazard ratio was 1.703 (95 per cent confidence interval, 1.373 to $2.112 ; z$ score $=4.85, p=0.000$ ) for overall survival and was 1.918 (95 per cent confidence interval, 1.410 to $2.609 ; z$ score $=4.15, p=0.000$ ) for disease-free survival. No significant publication bias was found. A sensitivity analysis showed that the results were robust. Power analyses also showed there was enough power to detect the calculated hazard ratios.

Conclusion: The study found that vascular endothelial growth factor overexpression predicted a worse prognosis for laryngeal cancer patients. This supports a strategy of targeted therapy by blocking the vascular endothelial growth factor receptor.
\end{abstract}

Key words: Laryngeal Neoplasms; Vascular Endothelial Growth Factors; Disease-Free Survival; Survival; Biological Markers

\section{Introduction}

According to the latest cancer statistics, there were 13560 cases of laryngeal cancer and 3640 laryngeal cancer deaths in the USA in $2015 .^{1}$ Globally, the age-standardised incidence of laryngeal cancer was 5.1 per 100000 and the age-standardised mortality rate was 2.2 per 100000 in more developed areas; these figures were 3.5 and 2.0 per 100000 , respectively, in less developed areas. ${ }^{2}$ In recent years, improvements in chemotherapy, radiotherapy and conservative surgery have led to improved preservation of organs and their functions, leading to a better quality of life for laryngeal cancer patients. ${ }^{3}$ However, patient prognosis has not improved: the 5-year relative survival rates for 1975-1977, 1987-1989 and 2004-2010 were 66 per cent, 66 per cent and 63 per cent, respectively. ${ }^{1}$ It is therefore important to identify prognosis factors for this disease and for developing personalised treatment.

Vascular endothelial growth factor controls tumour angiogenesis by promoting endothelial cell growth and migration. Previous studies found that vascular endothelial growth factor overexpression was associated with poor prognosis for many human cancers, including colorectal cancer, hepatocellular cancer, gastric cancer, cervical cancer and osteosarcoma. ${ }^{4-8}$ A meta-analysis found that vascular endothelial growth factor positivity was associated with worse overall survival for head and neck squamous cell carcinoma patients, with an estimated risk of death within 2 years of 1.88 (95 per cent confidence interval (CI), 1.43 to $2.45 ; p<0.001){ }^{9}$ Although the larynx is, strictly speaking, part of the head and neck region, it has several clinical and molecular peculiarities. A comparative genomic study identified differences in chromosomal patterns and cancer progression between laryngeal cancer and other head and neck cancers. ${ }^{10}$ The American Cancer Society classifies the larynx as part of the respiratory system, that is, as separate from the oral cavity and pharynx. The relationship between vascular endothelial growth factor expression and laryngeal cancer prognosis is unknown.

A meta-analysis was performed to summarise the scientific evidence for an association between vascular 
endothelial growth factor expression and the laryngeal cancer prognosis.

\section{Materials and methods}

Database and literature search

Systematic computerised searches of the Medline and Web of Science databases up to 31 January 2015 were performed. The following search terms were used: 'laryngeal carcinoma', 'laryngeal cancer', 'vascular endothelial growth factor', 'VEGF', 'prognosis', 'disease-free survival' and 'overall survival'. The search strategy for Medline was 'laryngeal neoplasms' [MeSH Terms] AND (vascular endothelial growth factor* [Title/Abstract] OR VEGF* [Title/Abstract]) AND (Survi* [Title/Abstract] OR prognosis [Title/ Abstract]). For Web of Science, the search strategy was $\left(\mathrm{Ts}=\mathrm{Laryn}^{*}\right)$ and $(\mathrm{Ts}=$ vascular endothelial growth factor* or Ts $\left.=\mathrm{VEGF}^{*}\right)$ and $\left(\mathrm{Ts}=\right.$ Survi $^{*}$ or $\mathrm{Ts}=$ prognosis). In addition, the references of all relevant studies were manually reviewed to supplement the search results.

\section{Study selection}

Relevant studies were manually selected based on the following criteria: (1) vascular endothelial growth factor expression was measured immunohistochemically or by enzyme-linked immunosorbent assay or other detection methods; (2) overall survival and disease-free survival were compared for different levels of vascular endothelial growth factor expression in laryngeal cancer; (3) hazard ratios and 95 per cent CIs for overall survival or disease-free survival stratified by the level of vascular endothelial growth factor expression were obtained by multivariate Cox proportional hazards regression or univariate Kaplan-Meier log-rank tests, or from the reported data or survival curves.

\section{Data extraction}

The following data types were extracted into standardised forms: (1) basic information such as the first author, publication year, country, study design and number of patients enrolled per year; (2) patient characteristics such as sex, age, clinical stage and treatment; (3) the vascular endothelial growth factor detection method; and (4) outcome, such as overall survival and disease-free survival.

Study selection and data extraction were carried out independently by two reviewers. Disagreements were resolved by discussion between the two.

\section{Statistical analysis}

Methods used to combine time-to-event outcomes were summarised using the log hazard ratio and its variance. ${ }^{11,12}$ If individual studies provided a hazard ratio but not a 95 per cent CI, the latter was calculated using the formulas: $b=\ln$ (hazard ratio); standard error of the mean $(\mathrm{SEM})=b \div$ inverse_normal_ distribution $(p \div 2)$; and 95 per cent $\mathrm{CI}=\exp (b \pm$ $1.96 \times \mathrm{SEM}$ ). If individual studies provided neither hazard ratios nor 95 per cent CIs, data were extracted from Kaplan-Meier survival curves using a previously reported method and hazard ratio calculations spreadsheet (in additional file 1 of the paper at http://www. ncbi.nlm.nih.gov/pmc/articles/PMC1920534/?tool= pubmed\#S1). ${ }^{13}$ Survival curves were read using Engauge Digitizer version 4.1 (http://digitizer.source forge.net/). Statistical heterogeneity was investigated using inconsistency $\left(I^{2}\right)$ statistics: an $I^{2}$ value of 50 per cent or more represented substantial heterogeneity. Studies without substantial heterogeneity were pooled using a fixed-effect model; studies with heterogeneous data were pooled using a random-effects model. Potential publication bias was estimated using a funnel graph, Egger's regression tests and Begg's adjusted rank correlation tests. A sensitivity analysis was conducted using only pooled adjusted hazard ratios. Meta-analyses were performed using Stata 12.0 (StataCorp, College Station, Texas, USA). As a limited number of studies was included in this metaanalysis, we also carried out power analyses. Statistical power was calculated using the power and sample size calculation in PS: Power and Sample Size Calculation version 3.0 (http://biostat.mc.vander bilt.edu/wiki/Main/PowerSampleSize).

\section{Results}

\section{Description of eligible studies}

In all, seven studies met our inclusion criteria for meta-analysis. ${ }^{14-20}$ The detailed steps in the literature search are shown in Figure 1. Data from 975 patients were used in pooled analyses; study sample sizes ranged from 59 to 289 . Two studies each were from USA, Spain and China, and the other was conducted in Greece. In six studies, patient treatments included surgery; in the other study, patients underwent primary radiotherapy only. Vascular endothelial growth factor expression was detected immunohistochemically in six studies and by enzyme-linked immunosorbent assay in the other. Overall follow up ranged from 60 months to 200 months. Six studies used univariate and multivariate analysis to evaluate prognostic outcomes; for these, adjusted hazard ratios were used in this meta-analysis. One study used only univariate analysis to evaluate prognostic outcomes; for this study, crude hazard ratios were used in the meta-analysis. Table I shows the main characteristics of the seven studies included in this meta-analysis.

\section{Overall survival}

Six studies reported overall survival as a prognostic outcome $^{14-18,20}$; there was no heterogeneity between these studies $\left(I^{2}=0.0\right.$ per cent, $\left.p=0.512\right)$. A pooled hazard ratio of 1.703 (95 per cent CI, 1.373 to 2.112; $z$ score $=4.85, p=0.000)$ was obtained using a fixed-effect model (Figure. 2). The symmetrical 


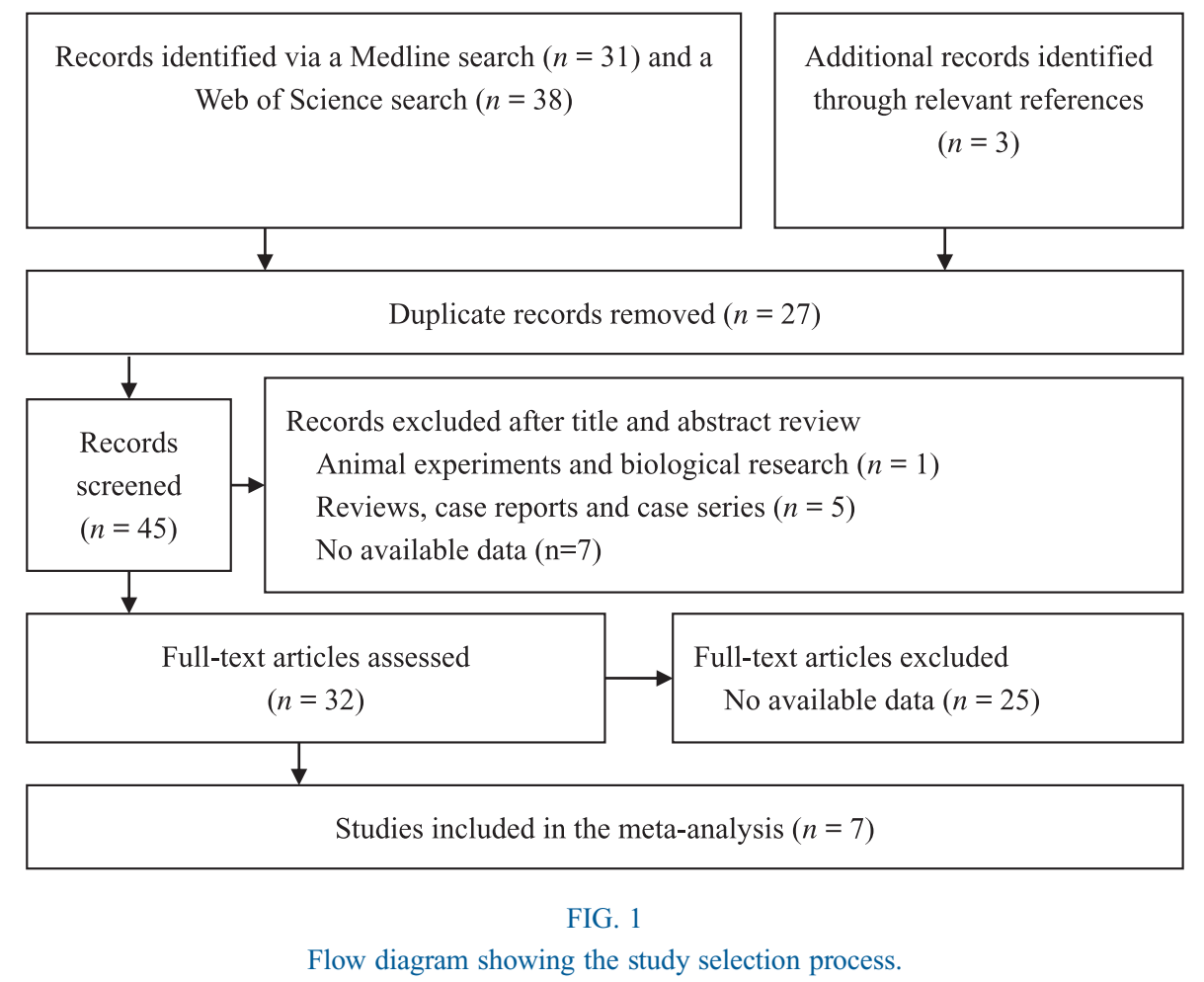

funnel plot (Figure 3 ) and the Begg's test $(z$ score $=$ $0.38, p=0.707)$ and Egger's test $(t=0.87, p=$ 0.432 ) suggested no significant publication bias. A sensitivity analysis using only adjusted hazard ratios found the pooled hazard ratio to be 1.688 (95 per cent $\mathrm{CI}, 1.35$ to 2.104; $z$ score $=4.66, p=0.000)$, indicating that the result was robust. A power analysis found that this sample had sufficient power (51.3 per cent) to detect a hazard ratio of 1.703 .

These results show that vascular endothelial growth factor overexpression was associated with worse overall survival for laryngeal cancer patients.

\section{Disease-free survival}

Five studies reported disease-free survival as a prognostic outcome; ${ }^{14,15,17-19}$ there was no heterogeneity between these studies $\left(I^{2}=0.0\right.$ per cent, $\left.p=0.512\right)$. A pooled hazard ratio of 1.918 ( 95 per cent $\mathrm{CI}, 1.410$ to $2.609 ; z$ score $=4.15, p=0.000)$ was obtained using a fixed-effect model (Figure. 4). The symmetrical funnel plot (Figure. 5), and the Begg's test ( $z$ score $=$ $0.24, p=0.806)$ and Egger's test $(t=0.55, p=$ $0.619)$ suggested no significant publication bias. A sensitivity analysis of pooled adjusted hazard ratios showed that the pooled hazard ratio was 1.938 (95 per cent $\mathrm{CI}, 1.405$ to $2.673 ; z$ score $=4.03, p=$ $0.000)$, indicating that the result is robust. A power analysis showed this sample had sufficient power (43.6 per cent) to detect a hazard ratio of 1.918 .

These results show that vascular endothelial growth factor overexpression was associated with a worse disease-free survival rate in laryngeal cancer patients.

\section{Discussion}

Vascular endothelial growth factor was first identified in 1983, and first purified and cloned in 1989. It functions as an endothelial cell-specific mitogen in vitro and as an angiogenic inducer in a variety of in vivo models. $^{21}$ This study showed that vascular endothelial growth factor overexpression predicts a worse prognosis for laryngeal cancer patients: the pooled hazard ratio was 1.703 (95 per cent CI, 1.373 to 2.112) for overall survival and 1.918 (95 per cent CI, 1.410 to 2.609) for disease-free survival. Several mechanisms could mediate the association between vascular endothelial growth factor overexpression and poor laryngeal cancer prognosis. Angiogenesis is important for the growth and progression of solid tumours, and vascular endothelial growth factor both induces mitosis and regulates the endothelial cell permeability. ${ }^{22}$ Vascular endothelial growth factor is also important for inducing immune tolerance in the tumour microenvironment. ${ }^{23}$ Deezagi et al. reported that small interfering RNAmediated vascular endothelial growth factor inhibition induces apoptosis in cancer cells. ${ }^{24}$ Therefore, promotion of angiogenesis and immune tolerance by vascular endothelial growth factor overexpression may worsen laryngeal cancer prognosis.

Other risk factors are also reported to affect laryngeal cancer prognosis: these include the tumour-node-metastasis (TNM) tumour category (as defined by the Union for International Cancer Control), nodal involvement (reported for both radiotherapeutic or surgical series), different locations (glottic tumours have a a more favourable prognosis than supraglottic or subglottic tumours), alcohol consumption, and the 


\begin{tabular}{|c|c|c|c|c|c|c|c|c|c|c|c|c|c|}
\hline \multicolumn{14}{|c|}{$\begin{array}{c}\text { TABLE I } \\
\text { CHARACTERISTICS OF STUDIES INCLUDED IN THE META-ANALYSIS }\end{array}$} \\
\hline Study & Year & Country & Design & Enrollment period & $\begin{array}{l}\text { Clinical } \\
\text { stage }\end{array}$ & $\begin{array}{l}\text { Pts } \\
(n)\end{array}$ & $\begin{array}{l}\text { Sex } \\
(M / F)\end{array}$ & Age (y) & Treatment & $\begin{array}{l}\text { VEGF } \\
\text { detection } \\
\text { method }\end{array}$ & FU (mon) & Outcomes & $\begin{array}{l}\text { Statistical } \\
\text { analysis } \\
\text { methods }\end{array}$ \\
\hline Liu et $a .^{14}$ & 2013 & China & Prospective & Jan 2000 - Oct 2005 & I-IV & 176 & $170 / 6$ & $<58.85,>58.91$ & $\begin{array}{l}\text { Partial or total } \\
\text { laryngectomy }\end{array}$ & $\mathrm{IHC}$ & $\sim 60$ & DFS \& OS & $\mathrm{U} \& \mathrm{M}$ \\
\hline $\begin{array}{l}\text { Pentheroudakis } \\
\text { et al. }{ }^{15}\end{array}$ & 2012 & Greece & Retrospective & May 1985 - Jun 2008 & I-III & 289 & $277 / 12$ & $63(36-82)$ & $\begin{array}{l}\text { Potentially curative } \\
\text { surgery and/or } \\
\text { radical external beam } \\
\text { irradiation }\end{array}$ & $\mathrm{IHC}$ & $\sim 200$ & DFS \& OS & $U \&$ M \\
\hline Liu et al. ${ }^{16}$ & 2011 & China & Prospective & Jan 2003 - Sep 2005 & I-IV & 69 & $66 / 3$ & $<58.29,>58.40$ & Laryngectomy & IHC & $\sim 60$ & OS & $\mathrm{U} \& \mathrm{M}$ \\
\hline Rueda et al. ${ }^{17}$ & 2011 & Spain & Retrospective & $1993-2003$ & III-IV & 59 & NR & 39-73 (median 58) & $\begin{array}{l}\text { Surgery, chemotherapy } \\
\& \text { radical } \\
\text { radiotherapy }\end{array}$ & IHC & $\sim 200$ & DFS \& OS & U only* \\
\hline Parikh et al..$^{18}$ & 2007 & USA & Retrospective & $1975-2000$ & $\mathrm{~T}_{1-2} \mathrm{~N}_{0}$ & 123 & $106 / 17$ & $\begin{array}{l}\text { Median } \\
\quad 64.06 \pm 10.9 \\
\text { range } 38-90\end{array}$ & $\begin{array}{l}\text { Primary radiation } \\
\text { therapy }\end{array}$ & $\mathrm{IHC}$ & $\begin{array}{l}\text { Median } 9.9 \\
\text { years }\end{array}$ & DFS \& OS & $U \& M$ \\
\hline $\begin{array}{l}\text { Hinojar-Gutiérrez } \\
\text { et al. }\end{array}$ & 2007 & Spain & Retrospective & NR & $\mathrm{T}_{1-4} \mathrm{~N}_{0}$ & 76 & $74 / 2$ & $\begin{array}{l}\text { median } 64 \text {, range } \\
41-87\end{array}$ & Surgery & IHC & $\begin{array}{l}\text { Median } 71 \\
\quad \text { (range } \\
40-128)\end{array}$ & DFS & $\mathrm{U} \& \mathrm{M}$ \\
\hline Teknos et al..$^{20}$ & 2002 & USA & Prospective & NR & III-IV & 183 & $179 / 4$ & NR & $\begin{array}{l}\text { Surgery, chemotherapy } \\
\& \text { radical } \\
\text { radiotherapy }\end{array}$ & ELISA & $\sim 80$ & OS & $U \& M$ \\
\hline
\end{tabular}


Liu et al. ${ }^{14}$

Pentheroudakis et al. ${ }^{15}$

Liu et al. ${ }^{16}$

Rueda et al. ${ }^{17}$

Parikh et al. ${ }^{18}$

Teknos et al. ${ }^{20}$

Overall $\left(I^{2}=0.0 \%, p=0.512\right)$

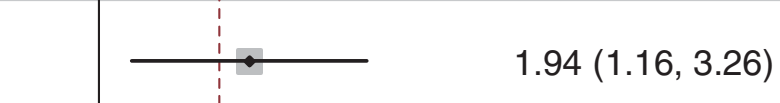

$1.54(1.02,2.33)$

$1.32(0.69,2.53)$

$2.06(0.76,5.91)$

$2.79(1.49,4.95)$

$1.47(0.98,2.23)$

27.30

$1.70(1.37,2.11)$

100.00

FIG. 2

Meta-analysis of overall survival in laryngeal squamous cell carcinoma patients with different levels of vascular endothelial growth factor expression. $\mathrm{HR}=$ hazard ratio; $\mathrm{CI}=$ confidence interval

expression of genes such as $B C L 2, p 53$ and $B A X .^{25}$ These prognostic factors are confounders in the assessment of the relationship between vascular endothelial growth factor expression and laryngeal cancer prognosis. This study included seven studies, of which six reported multivariate analyses and hazard ratios adjusted by sex, age, smoking history, alcohol intake, histological grade, primary tumour site, TNM tumour classification, clinical stage, the presence of metastasis and the expression of some tumour markers. A sensitivity analysis of pooled adjusted hazard ratios showed that the results were robust.

The results of this study support a therapeutic strategy of blocking the vascular endothelial growth factor receptor. Several clinical trials have confirmed the effectiveness of this type of therapy. In a prospective trial, Yoo et al. treated locally advanced head and neck cancer with bevacizumab, erlotinib and concurrent chemoradiation. ${ }^{26}$ After a median follow up of 46 months, they reported 3-year locoregional control

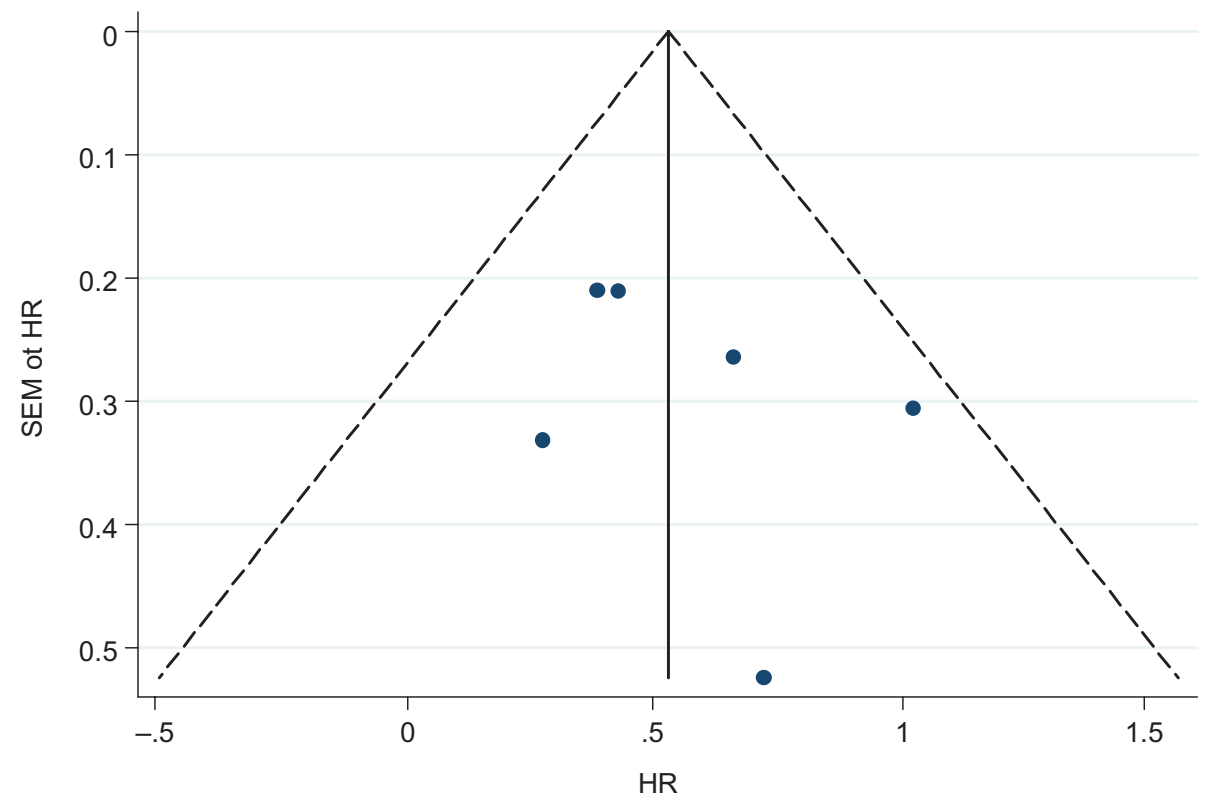

FIG. 3

Funnel plot showing an analysis of publication bias in the pooled hazard ratios for overall survival. SEM = standard error of the mean; $\mathrm{HR}=$ hazard ratio 
Liu et al. ${ }^{14}$

Pentheroudakis et al. ${ }^{15}$

Rueda et al. ${ }^{17}$

Parikh et al. ${ }^{18}$

Hinojar-Gutiérrez et al.

Overall $\left(I^{2}=0.0 \%, p=0.512\right)$

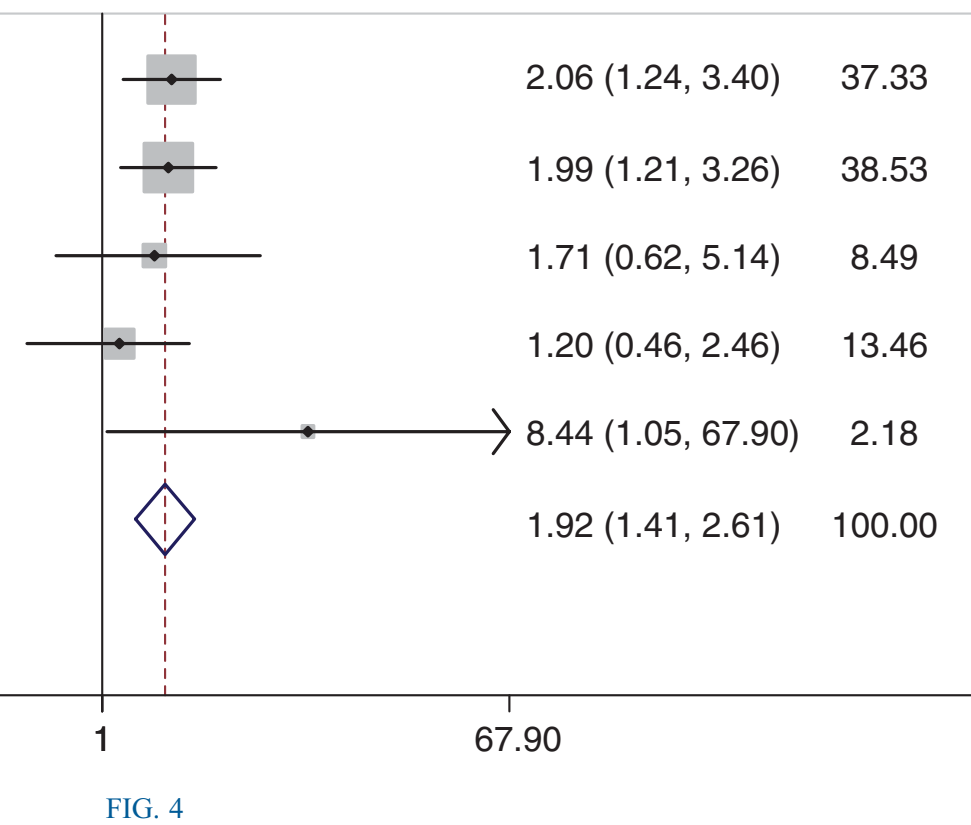

FIG. 4

Meta-analysis of progression-free survival in laryngeal squamous cell carcinoma patients with different levels of vascular endothelial growth factor expression. $\mathrm{HR}=$ hazard ratio; $\mathrm{CI}=$ confidence interval

and distant metastasis-free survival rates of 85 per cent and 93 per cent, respectively, and 3-year estimated progression-free survival, disease-specific survival and overall survival rates of 82 per cent, 89 per cent and 86 per cent, respectively. In a phase II trial by Hainsworth et al., combined modality treatment comprising chemotherapy, radiotherapy, bevacizumab and erlotinib in patients with locally advanced squamous carcinoma of the head and neck resulted in estimated three-year progression-free and overall survival rates of 71 per cent and 82 per cent, respectively. ${ }^{27}$ The clinical data showed acceptable toxicity levels. This meta-analysis had some limitations. Firstly, some of the studies included failed to provide sufficient data on time-to-event outcomes for direct inclusion in the meta-analysis. Further, data extracted from survival curves was used instead of direct patient data, which may have led to inaccuracies. Secondly, the studies included different clinical tumour stages, different treatments and different methods of detecting vascular endothelial growth factor. Therefore, a subgroup analysis would have been preferable. However, this was

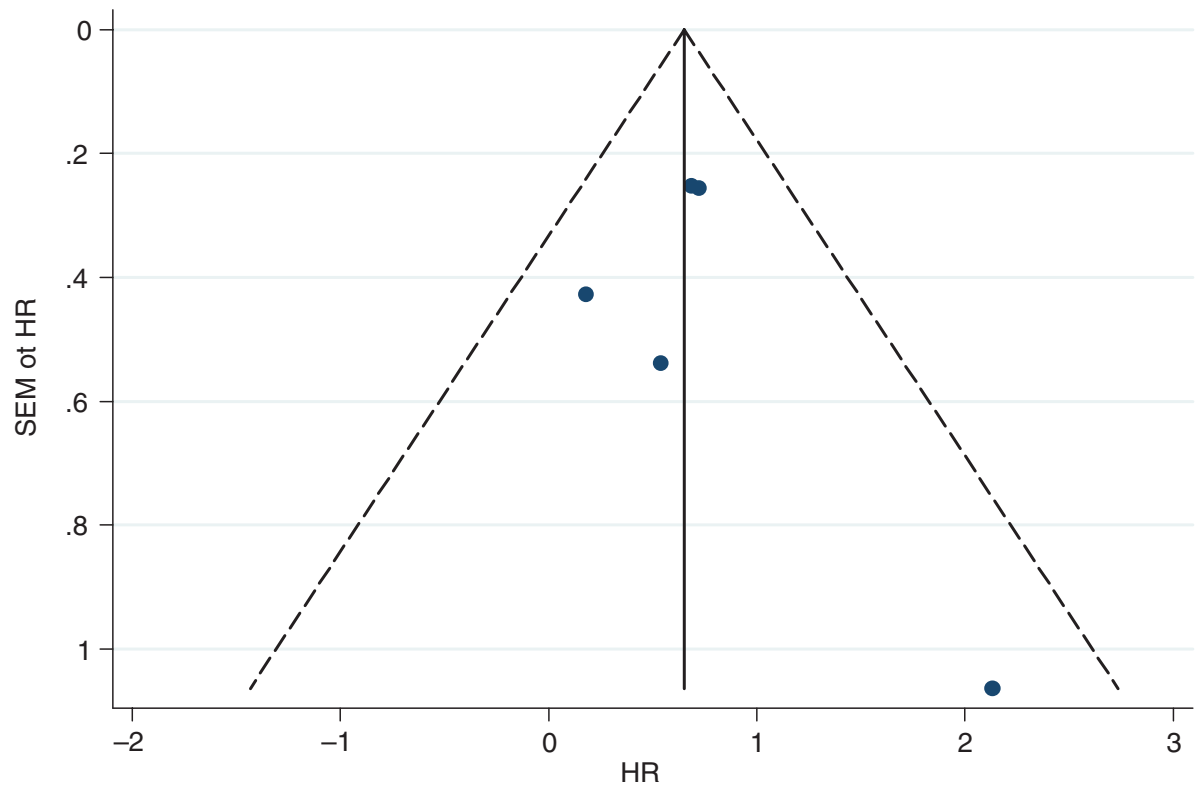

FIG. 5

Funnel plot showing an analysis of publication bias in the pooled hazard ratios for progression-free survival. SEM = standard error of the mean; $\mathrm{HR}=$ hazard ratio 
impossible owing to the limited number of studies included in the meta-analysis. Fortunately, there was no heterogeneity between studies.

- Vascular endothelial growth factor is an angiogenic factor that promotes endothelial cell growth and migration

- Outcomes derived from the pooled data of 7 studies involving 975 laryngeal cancer patients were included in a meta-analysis

- Vascular endothelial growth factor overexpression predicted a worse prognosis

- Pooled hazard ratios for overall survival and disease-free survival were 1.703 (95 per cent CI, 1.373 to 2.112) and 1.918 (95 per cent CI, 1.410 to 2.609 ), respectively

\section{Conclusion}

This meta-analysis found that vascular endothelial growth factor overexpression predicts a worse prognosis for laryngeal cancer patients. This evidence supports a strategy of targeted therapy by blocking the vascular endothelial growth factor receptor.

References

1 Siegel RL, Miller KD, Jemal A. Cancer statistics, 2015. CA Cancer J Clin 2015;65:5-29

2 Torre LA, Bray F, Siegel RL, Ferlay J, Lortet-Tieulent J, Jemal A. Global cancer statistics, 2012. CA Cancer J Clin 2015;65: 87-108

3 Jenckel F, Knecht R. State of the art in the treatment of laryngeal cancer. 2013;33:4701-10

4 Kim NK, Park JK, Shin E, Kim YW. The combination of nuclear factor kappa B, cyclo-oxygenase-2 and vascular endothelial growth factor expression predicts poor prognosis in stage II and III colorectal cancer. Anticancer Res 2014;34:6451-7

5 Li T, Zhu Y, Ren W, Xu S, Yang Z, Fang A et al. High coexpression of vascular endothelial growth factor receptor-1 and Snail is associated with poor prognosis after curative resection of hepatocellular carcinoma. Med Oncol 2012;29:2750-61

6 He W, Tang B, Yang D, Li Y, Song W, Cheang T et al. Doublepositive expression of high-mobility group box 1 and vascular endothelial growth factor $\mathrm{C}$ indicates a poorer prognosis in gastric cancer patients. World J Surg Oncol 2013; 18:161

7 DeMei M, XiangXin L, YongPing X, YongXia Y, YunHai Y, Lin Z. Vascular endothelial growth factor $C$ expression is closely correlated with lymph node recurrence and poor prognosis in patients with early stage cervical cancer. J Int Med Res 2013;41:1541-9

8 Chen D, Zhang YJ, Zhu KW, Wang WC. A systematic review of vascular endothelial growth factor expression as a biomarker of prognosis in patients with osteosarcoma. Tumour Biol 2013;34: 1895-9

9 Kyzas PA, Cunha IW, Ioannidis JP. Prognostic significance of vascular endothelial growth factor immunohistochemical expression in head and neck squamous cell carcinoma: a meta-analysis. Clin Cancer Res 2005;11:1434-40

10 Huang Q, Yu GP, McCormick SA, Mo J, Datta B, Mahimkar M et al. Genetic differences detected by comparative genomic hybridization in head and neck squamous cell carcinomas from different tumor sites: construction of oncogenetic trees for tumor progression. Genes Chromosomes Cancer 2002;34:224-33

11 Parmar M, Torri V, Stewart L. Extracting summary statistics to perform meta-analyses of the published literature for survival endpoints. Stat Med 1998;17:2815-34

12 Williamson RP, Smith TC, Hutton JL, Marson AG. Aggregate data meta-analysis with time-to-event outcome. Stat Med 2002;21:3337-51
13 Tierney JF, Stewart LA, Ghersi D, Burdett S, Sydes MR. Practical methods for incorporating summary time-to-event data into meta-analysis. Trials 2007;8:16

14 Liu Y, Su Z, Li G, Yu C, Ren S, Huang D et al. Increased expression of metadherin protein predicts worse disease-free and overall survival in laryngeal cancer. Int J Cancer 2013; 133:671-9

15 Pentheroudakis G, Nicolaou I, Kotoula V, Fountzilas E, Markou $\mathrm{K}$, Eleftheraki AG et al. Prognostic utility of angiogenesis and hypoxia effectors in patients with operable squamous cell cancer of the larynx. Oral Oncol 2012;48:709-16

16 Liu Y, Qiu Y, Zhang X, Tian Y, Huang D, Zhou X. The expression and correlation of HMGB1 and VEGF protein in laryngeal cancer. Lin Chung Er Bi Yan Hou Tou Jing Wai Ke Za Zhi 2011; 25:265-9

17 Rueda A, Cazorla O, Pérez L, Álvarez M, Redondo M, Gallego E et al. Vascular endothelial growth factor and vascular endothelial growth factor receptor-2 tumor expression in patients with advanced laryngeal cancer after induction chemotherapy for organ preservation. Head Neck 2011;33:808-16

18 Parikh RR, Yang Q, Haffty BG. Prognostic significance of vascular endothelial growth factor protein levels in T1-2 N0 laryngeal cancer treated with primary radiation therapy. Cancer 2007; 109:566-73

19 Hinojar-Gutiérrez A, Fernández-Contreras ME, GonzálezGonzález R, Fernández-Luque MJ, Hinojar-Arzadún A, Quintanilla $\mathrm{M}$ et al. Intratumoral lymphatic vessels and VEGF-C expression are predictive factors of lymph node relapse in T1T4 N0 laryngopharyngeal squamous cell carcinoma. Ann Surg Oncol 2007;14:248-57

20 Teknos TN, Cox C, Yoo S, Chepeha DB, Wolf GT, Bradford CR et al. Elevated serum vascular endothelial growth factor and decreased survival in advanced laryngeal carcinoma. Head Neck 2002;24:1004-11

21 Ferrara N. Vascular endothelial growth factor: basic science and clinical progress. Endocr Rev 2004;25:581-611

22 Roskoski R Jr. Vascular endothelial growth factor (VEGF) signaling in tumor progression. Crit Rev Oncol Hematol 2007;62: 179-213

23 Johnson BF, Clay TM, Hobeika AC, Lyerly HK, Morse MA. Vascular endothelial growth factor and immunosuppression in cancer: current knowledge and potential for new therapy. Expert Opin Biol Ther 2007;7:449-60

24 Deezagi A, Ansari-Majd S, Vaseli-Hagh N. Induced apoptosis in human prostate cancer cells by blocking of vascular endothelial growth factor by siRNA. Clin Transl Oncol 2012;14:791-9

25 Licitra L, Bernier J, Grandi C, Locati L, Merlano M, Gatta G et al. Cancer of the larynx. Crit Rev Oncol Hematol 2003;47: 65-80

26 Yoo DS, Kirkpatrick JP, Craciunescu O, Broadwater G, Peterson $\mathrm{BL}$, Carroll MD et al. Prospective trial of synchronous bevacizumab, erlotinib, and concurrent chemoradiation in locally advanced head and neck cancer. Clin Cancer Res 2012;18:1404-14

27 Hainsworth JD, Spigel DR, Greco FA, Shipley DL, Peyton J, Rubin $\mathrm{M}$ et al. Combined modality treatment with chemotherapy, radiation therapy, bevacizumab, and erlotinib in patients with locally advanced squamous carcinoma of the head and neck: a phase II trial of the Sarah Cannon oncology research consortium. Cancer J 2011;17:267-72

Address for correspondence:

Dr L-P Zhang,

Department of Otolaryngology Head and Neck Surgery,

Affiliated Hospital of Nantong University,

20 Xi Si Road,

Nantong city,

Jiangsu,

226001, PR China

E-mail: pphss@126.com

Dr L-P Zhang takes responsibility for the integrity of the content of the paper

Competing interests: None declared 